\title{
Optical conductivity of multifold fermions: The case of RhSi
}

\author{
L. Z. Maulana $\odot,{ }^{1}$ K. Manna $\odot,{ }^{2}$ E. Uykur $\odot,{ }^{1}$ C. Felser $\odot,{ }^{2}$ M. Dressel $\odot,{ }^{1}$ and A. V. Pronin $\oplus^{1, *}$ \\ ${ }^{1}$ Physikalisches Institut, Universität Stuttgart, 70569 Stuttgart, Germany \\ ${ }^{2}$ Max-Planck-Institut für Chemische Physik fester Stoffe, 01187 Dresden, Germany
}

(Received 26 November 2019; accepted 12 March 2020; published 8 April 2020)

\begin{abstract}
We measured the reflectivity of the multifold semimetal $\mathrm{RhSi}$ in a frequency range from 80 to $20000 \mathrm{~cm}^{-1}$ $(10 \mathrm{meV}-2.5 \mathrm{eV})$ at temperatures down to $10 \mathrm{~K}$. The optical conductivity, calculated from the reflectivity, is dominated by the free-carrier (Drude) contribution below $1000 \mathrm{~cm}^{-1}(120 \mathrm{meV})$ and by interband transitions at higher frequencies. The temperature-induced changes in the spectra are generally weak: only the Drude bands narrow upon cooling, with an unscreened plasma frequency that is constant with temperature at approximately $1.4 \mathrm{eV}$, in agreement with a weak temperature dependence of the free-carrier concentration determined by Hall measurements. The interband portion of conductivity exhibits two linear-in-frequency regions below $5000 \mathrm{~cm}^{-1}$ $(\sim 600 \mathrm{meV})$, a broad flat maximum at around $6000 \mathrm{~cm}^{-1}(750 \mathrm{meV})$, and a further increase starting around $10000 \mathrm{~cm}^{-1}(\sim 1.2 \mathrm{eV})$. We assign the linear behavior of the interband conductivity to transitions between the linear bands near the band crossing points. Our findings are in accord with the predictions for the low-energy conductivity behavior in multifold semimetals and with earlier computations based on band structure calculations for RhSi.
\end{abstract}

DOI: 10.1103/PhysRevResearch.2.023018

\section{INTRODUCTION}

Multifold fermions are quasiparticles described by higherspin generalizations of the Weyl equation. They can be realized in the multifold semimetals: materials which possess characteristic electronic band crossings with degeneracies higher than 2 [1,2]. A number of such semimetals were recently predicted and experimentally confirmed among the materials from the space group 198 (SG198), whose symmetry is noncentrosymmetric and has no mirror planes, leading to a realization of "topological chiral crystals" [3-9]. In such semimetals, the quantized circular photogalvanic effect (QCPGE) was forecasted in 2017 [10]. In this nonlinear optical phenomenon, helical (i.e., circularly polarized) photons excite the chiral band carriers in such a way that the resultant photocurrent is quantized in units of material-independent fundamental constants. Recently, the observation of QCPGE was reported in $\mathrm{RhSi}$ [11], a member of SG198 and an established multifold semimetal [4-6].

These remarkable theoretical results and experimental observations demand further optical characterizations of $\mathrm{RhSi}$, in particular since the knowledge of frequency-dependent conductivity, $\sigma(\omega)=\sigma_{1}(\omega)+i \sigma_{2}(\omega)$, is essential for proper interpretation of QCPGE experiments. Because there is no characteristic energy scale, the quasiparticles near the linearband crossings in three dimensions are expected to be

\footnotetext{
*artem.pronin@pi1.physik.uni-stuttgart.de

Published by the American Physical Society under the terms of the Creative Commons Attribution 4.0 International license. Further distribution of this work must maintain attribution to the author(s) and the published article's title, journal citation, and DOI.
}

characterized by an interband $\sigma_{1}(\omega)$ that is proportional to the probing light frequency [12-14]. Indeed, such linear $\sigma_{1}(\omega)$ has been observed in many Dirac and Weyl semimetals [15-18]. The optical conductivity of the multifold semimetals has also been predicted to demonstrate a linear $\sigma_{1}(\omega)$ [19]. Most recently, optical conductivity was specifically calculated for RhSi from its band structure [20]. In this paper, we experimentally examine these theoretical results.

\section{EXPERIMENT}

Single crystals of $\mathrm{RhSi}$ were grown in the same way as described in Ref. [11]. The vertical Bridgman crystal-growth technique was utilized to grow the crystals from the melt using a slightly off-stoichiometric composition (excess Si). First, a polycrystalline ingot was prepared using the arc-melt technique by mixing the stoichiometric amount of constituent $\mathrm{Rh}$ and Si elements of $99.99 \%$ purity. Then the crushed powder was filled in a custom-designed sharp-edged alumina tube and finally sealed inside a tantalum tube with argon atmosphere. A critical composition with slightly excess Si was maintained to ensure a flux growth inside the Bridgman ampule. The whole assembly was heated to $1550{ }^{\circ} \mathrm{C}$ with a rate of $200{ }^{\circ} \mathrm{C}$ per hour and halted for 12 hours to ensure good mixing of the liquid. Then the crucible was slowly pulled to $\sim 1100^{\circ} \mathrm{C}$ with a rate of $0.8 \mathrm{~mm} / \mathrm{h}$ and finally quenched to room temperature. The temperature profile was controlled by attaching a thermocouple at the bottom of the tantalum ampule containing the sample. Single crystals with average linear dimensions of a few millimeters were obtained. The crystals were first analyzed with a white beam backscattering Laue x-ray diffractometer at room temperature. The obtained single and sharp Laue spot could be indexed by a single pattern, revealing excellent quality of the grown crystals without any twinning 


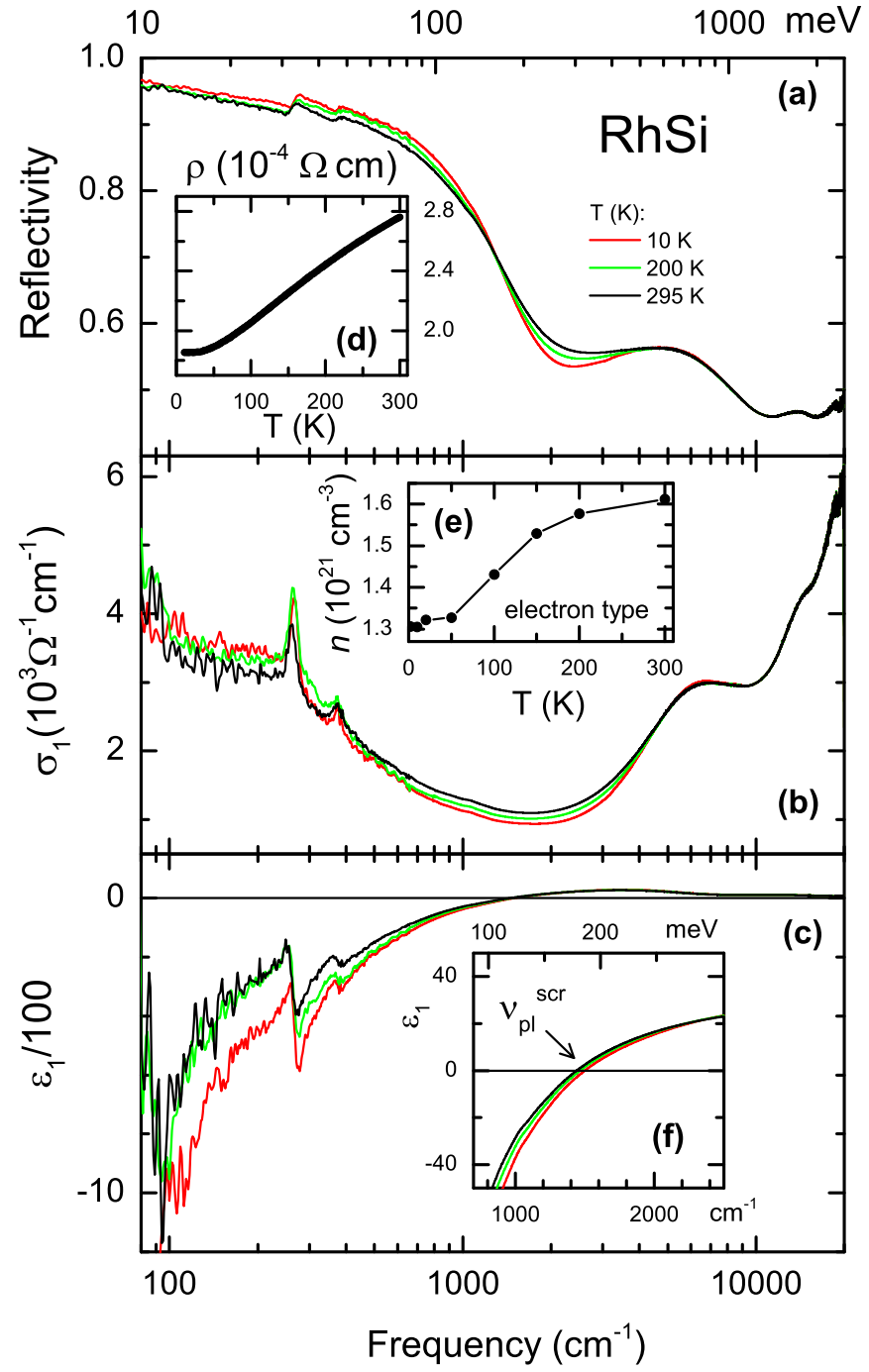

FIG. 1. Optical reflectivity (a) and the real parts of the optical conductivity (b) and the dielectric permittivity (c) of $\mathrm{RhSi}$ at $T=10$, 200, and 295 K. Note the logarithmic $x$ scale. The insets show: the dc resistivity vs. $T$ (d), the Hall electron concentration vs. $T$ (e), and a zoom of the permittivity spectra near the zero crossings (f).

or domains. The structural parameters were determined using a Rigaku AFC7 four-circle diffractometer with a Saturn 724+ CCD detector applying graphite-monochromatized Mo- $K \alpha$ radiation. The crystal structure was refined to be cubic $P 2{ }_{1} 3$ (SG198) with the lattice parameter $a=4.6858$ (9) $\AA$.

Temperature-dependent transport measurements (longitudinal de resistivity and Hall) were performed in a custommade setup at temperatures down to $2 \mathrm{~K}$. The results of these measurements are displayed in Figs. 1(d) and 1(e). A metallic behavior with a residual resistivity of $1.86 \times 10^{-4} \Omega \mathrm{cm}$ was observed. The Hall measurements evidenced electron conduction with a moderate increase of carrier concentration upon increasing temperature.

Optical reflectivity, $R(v)$, was measured on a polished surface [21] of a RhSi single crystal (with roughly $1.5 \times 1.5 \mathrm{~mm}^{2}$ in lateral dimensions) over a broad frequency range from $v=\omega /(2 \pi)=80$ to $20000 \mathrm{~cm}^{-1}(10 \mathrm{meV}-2.5 \mathrm{eV})$ at several different temperatures $(T=10,25,50,75,100,150,200$,

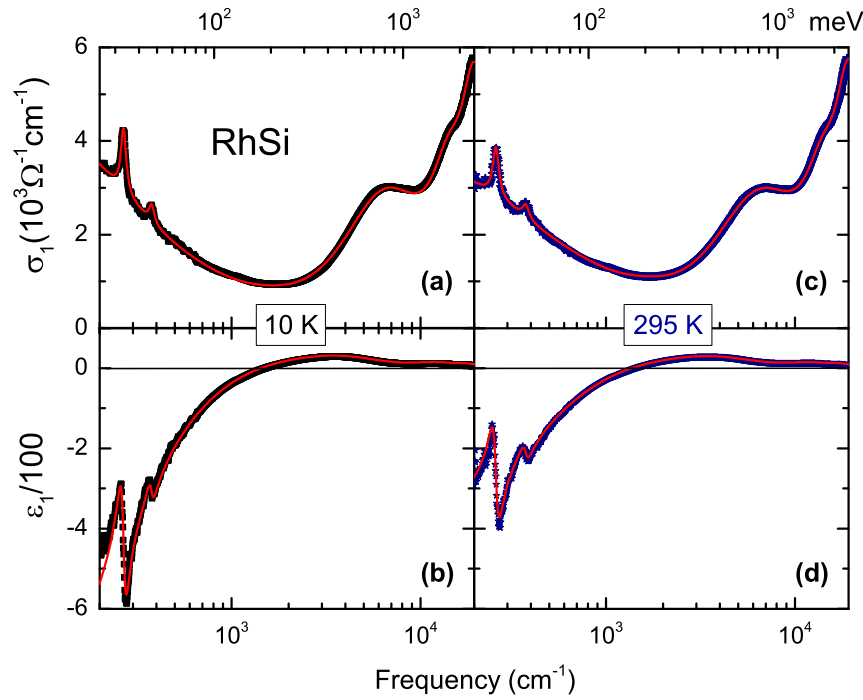

FIG. 2. Drude-Lorentz fits (lines) of the measured optical spectra (symbols) of $\sigma_{1}$ and $\varepsilon_{1}$ of $\mathrm{RhSi}$ at 10 and $295 \mathrm{~K}$, as indicated. Note the logarithmic $x$ scale.

$250,295 \mathrm{~K}$ ). The spectra in the far infrared (below $700 \mathrm{~cm}^{-1}$ ) were collected with a Bruker IFS 113v Fourier-transform spectrometer using in situ gold coating of the sample surface for reference measurements. At higher frequencies, a Bruker Hyperion infrared microscope attached to a Bruker Vertex $80 \mathrm{v}$ Fourier-transform infrared spectrometer was used. For these measurements, freshly evaporated gold mirrors served as reference. No sample anisotropy was detected in agrement with the cubic crystallographic structure.

For Kramers-Kronig analysis, zero-frequency extrapolations were made using the Hagen-Rubens relation in accordance with the temperature-dependent longitudinal dc resistivity measurements. For high-frequency extrapolations, we utilized the x-ray atomic scattering functions [22] followed by the free-electron behavior, $R(\omega) \propto 1 / \omega^{4}$, above $30 \mathrm{keV}$.

We found that the skin depth of the probing radiation exceeds $30 \mathrm{~nm}$ for all temperatures and frequencies (in the far-infrared range, it is above $200 \mathrm{~nm}$ ). Hence, our optical measurements reflect the bulk properties of $\mathrm{RhSi}$.

\section{RESULTS AND DISCUSSION}

Figure 1 displays the optical reflectivity $R(v)$ and the real parts of the optical conductivity and dielectric constant, $\varepsilon_{1}(v)=1-2 \sigma_{2}(v) / v$, for the studied RhSi sample in the full measurement-frequency range at three different temperatures. The overall temperature evolution of the spectra is minor. For frequencies higher than $\sim 8000 \mathrm{~cm}^{-1}(\sim 1 \mathrm{eV})$, the optical properties are fully independent of temperature. For the spectra analysis, we performed standard Drude-Lorentz fits [23], where the Drude terms describe the free-carrier response, while the Lorentzians mimic the interband optical transitions and phonons. Examples of such fits are presented in Fig. 2. Here we kept the zero-frequency limit of optical conductivity equal to the measured dc-conductivity value at every temperature. No other restrictions on the fit parameters were imposed. 
For best possible model description, the experimental spectra of $R(v), \sigma_{1}(v)$, and $\varepsilon_{1}(v)$ were fitted simultaneously.

\section{A. Electronic response}

At low frequencies, $\mathrm{RhSi}$ demonstrates a typical (semi)metallic response. The intraband contribution to the spectra can be best fitted with two Drude components, which have different scattering rates. Such multi-component Drude fits are often used to describe the optical response of multiband systems, particularly different semimetals [24-26]. In the case of $\mathrm{RhSi}$, the two-Drude approach can be justified by the presence of a few bands crossing the the Fermi level $[4,5,20,27]$; see Fig. 4(a): one set of bands is around the $R$ point and the others are near the $\Gamma$ and $M$ points of the Brillouin zone. The first set provides the dominating contribution to the free-carrier response and is also responsible for the electron type of conduction. Still, the second Drude term is necessary to describe the optical spectra accurately. Let us note that exact interpretation of such a two-Drude approach is arguable. The two components are not necessarily associated with two different bands, but might instead be related to the scattering processes within a band and between two different bands with a phonon involved.

The scattering rates of the Drude terms are found to be $250 \mathrm{~cm}^{-1}(\sim 30 \mathrm{meV})$ and $800 \mathrm{~cm}^{-1}(100 \mathrm{meV})$ at $100 \mathrm{~K}$. These values correspond to the midranges for the spectra at all measurement temperatures. At $T$ different from $100 \mathrm{~K}$, the scattering rates change only within $\pm 20 \%$ of these values. The midrange relaxation times are, hence, 21 and 6.6 fs. These values are somewhat larger than the value reported in another optical study of RhSi [11], indicating the improved quality of $\mathrm{RhSi}$ samples investigated in this work.

At around $1500 \mathrm{~cm}^{-1}(\sim 200 \mathrm{meV})$, a characteristic plasma edge is observed in $R(v)$. This edge correlates with the zero crossing of $\varepsilon(v)$, which corresponds to the screened plasma frequency, $v_{\mathrm{pl}}^{\mathrm{scr}}=v_{\mathrm{pl}} / \sqrt{\varepsilon_{\mathrm{inf}}}$. Here, $\varepsilon_{\mathrm{inf}}=65 \pm 5$, is the cumulative contribution of the higher-frequency optical transitions to $\varepsilon_{1}$ as obtained from the fits and $v_{\mathrm{pl}}$ is the unscreened plasma frequency. As best seen from Fig. 1(f), the screened plasma frequency is $v_{\mathrm{pl}}^{\text {scr }}=(1470 \pm 30) \mathrm{cm}^{-1}$ $\left[\hbar \omega_{\mathrm{pl}}^{\mathrm{scr}}=(182 \pm 4) \mathrm{meV}\right]$ and is independent of temperature, the corresponding unscreened plasma frequency being $v_{\mathrm{pl}}=$ $(11900 \pm 700) \mathrm{cm}^{-1}\left[\hbar \omega_{\mathrm{pl}}=(1470 \pm 90) \mathrm{meV}\right]$. This value of plasma frequency coincides within the experimental uncertainty with the value obtained from the Drude fits, $v_{\mathrm{pl}}=$ $(11200 \pm 600) \mathrm{cm}^{-1}\left[\hbar \omega_{\mathrm{pl}}=(1390 \pm 80) \mathrm{meV}\right]$, which includes contributions from both Drude terms and also shows no $T$ dependence.

The absence of any detectable temperature dependence of $\omega_{\mathrm{pl}}$ is in qualitative agreement with the very modest temperature-induced change of the carrier concentration: $n(T)$ increases by only $20 \%$ as $T$ goes from 2 to $300 \mathrm{~K}$; see Fig. 1(e).

The relatively large value of $\omega_{\mathrm{pl}}$ (cf. the results for other nodal semimetals $[15-18,28]$ ) and the fairly high $\left(\sim 10^{21} \mathrm{~cm}^{-3}\right)$ free-electron density of RhSi [see Fig. 1(d)] are consistent with the results of band structure calculations $[4,5,20,27]$, which show that the Fermi level in $\mathrm{RhSi}$ is quite deep in the conduction band for the electron momenta

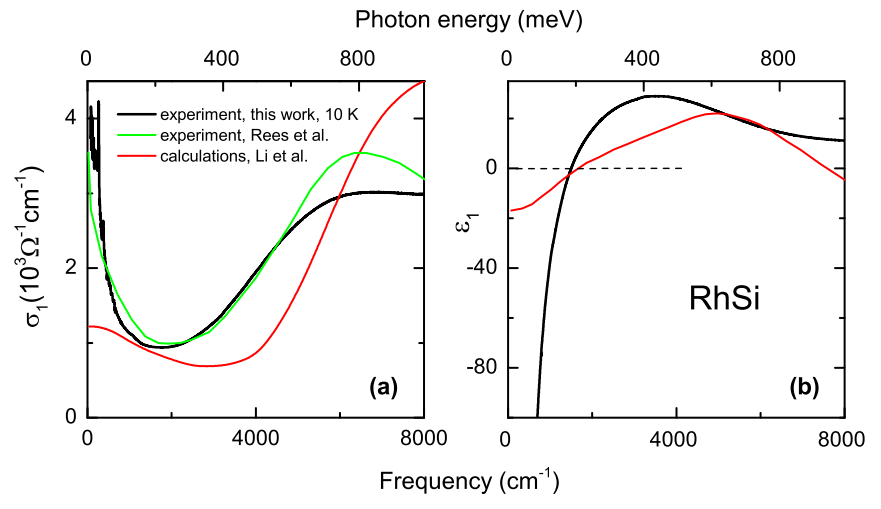

FIG. 3. Comparison of the experimental (this work and Ref. [11]) and calculated (Ref. [20]) optical spectra of RhSi: optical conductivity (a) and dielectric function (b).

near the corners ( $R$ points) of the Brillouin zone [Fig. 4(a)]. This situation makes the optical response of $\mathrm{RhSi}$ similar to the one observed in the Dirac semimetal $\mathrm{Au}_{2} \mathrm{~Pb}$ [29]: free carriers dominate the low-frequency $\left(v<2000 \mathrm{~cm}^{-1}\right.$, $\hbar \omega<250 \mathrm{meV})$ regions of $\sigma_{1}(v)$ and $\varepsilon(v)$. Still, unlike the situation in $\mathrm{Au}_{2} \mathrm{~Pb}$, the optical transitions between the linearly dispersing bands can be resolved in RhSi.

In Fig. 3 our optical findings at $T=10 \mathrm{~K}$ are plotted together with the results of band-structure-based calculations from Ref. [20] and with the previously reported measurements of Ref. [11]. The experimental curves follow each other quite well. The deviations between the curves can be explained by different free-carrier contributions (cf. the difference in the scattering times discussed above) and probably by a somewhat more accurate Kramers-Kronig analysis utilized in the present work: our reflectivity measurements are performed in a broader frequency range as compared to the measurements from Ref. [11].

Despite some discrepancy between the calculations and both experimental curves in Fig. 3(a), the match can be considered satisfactory. One has to keep in mind that calculations of the optical conductivity from the electronic band structure are rather challenging, particularly for semimetals: a survey of the available literature reveals only a qualitative match between the calculated optical conductivity and experimental results for a wide range of nodal semimetals studied recently $[18,25,30-32]$. Nevertheless, both the lowenergy features of the interband experimental $\sigma(v)$-the initial (i.e., for the frequencies just above the Drude rolloff) linear increase and the further flattening-are reproduced by theory.

In order to establish a better connection between the features observed in the most interesting, low-energy part of the experimental conductivity and the interband optical transitions, we show our $\sigma(v)$ together with the low-energy band structure of $\mathrm{RhSi}$ in Fig. 4. Additionally, we plot the interband contribution to the optical conductivity, $\sigma_{1}^{\text {inter }}(v)$, obtained by subtracting the Drude fits and the sharp phonon peaks (discussed below) from the measured spectra. The interband optical conductivity found this way is pretty much linear in frequency for $v<3000 \mathrm{~cm}^{-1}$. We found that this approximate linearity is robust: varying the fit parameters 

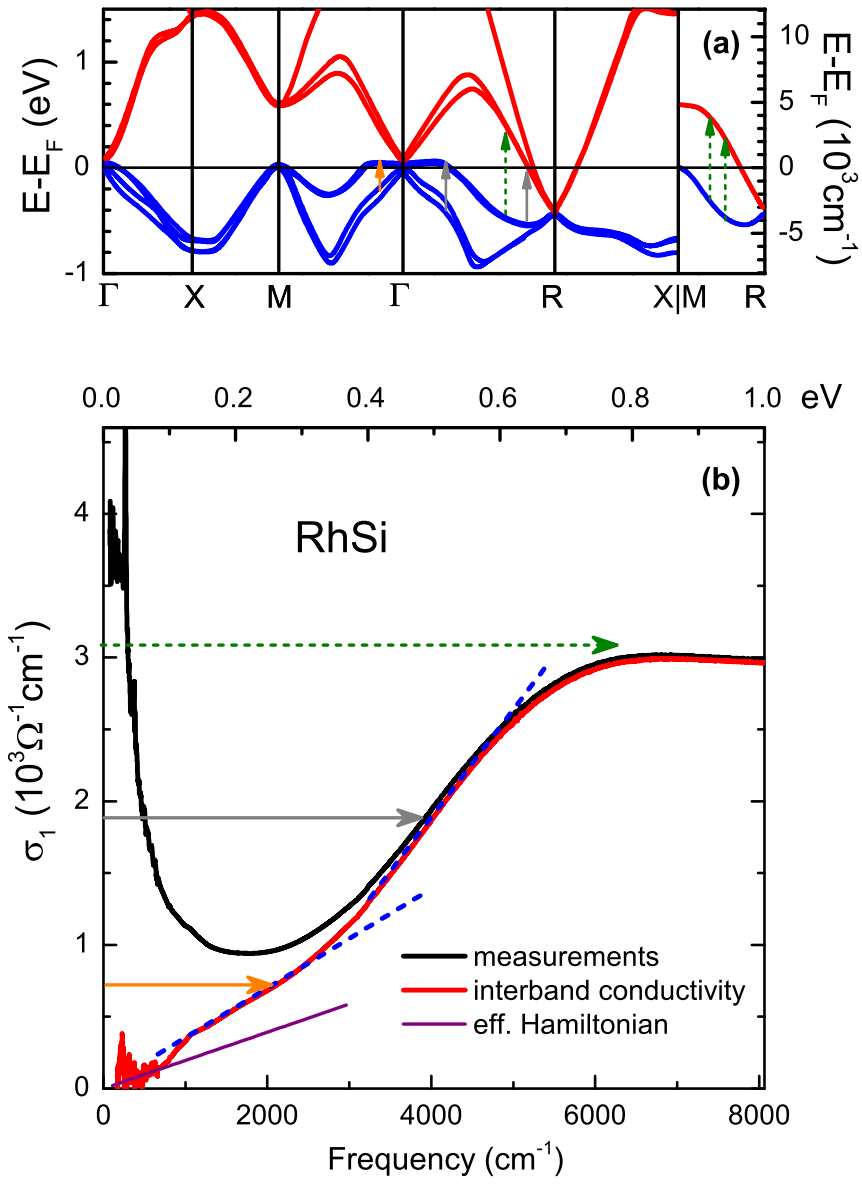

FIG. 4. Low-energy electronic structure of RhSi (a) and its optical conductivity (b). The electronic structure is calculated within the Topological Materials Database project [27]. Note that the band color code follows this reference and is not always related to the band position relative to the Fermi level. Both the as-measured conductivity spectra and the spectra after subtraction of the freecarrier response, $\sigma_{1}^{\text {inter }}(v)$, are shown in (b). The optical transitions responsible for the characteristic features in $\sigma_{1}^{\text {inter }}(v)$ are depicted as vertical arrows in (a). The corresponding frequency scales are indicated by the horizontal arrows of the same color in (b). The solid orange arrows represent the transitions between the approximately linear bands (including the almost flat bands) near the $\Gamma$ point and the corresponding linear $\sigma_{1}^{\text {inter }}(v)$. The solid grey arrows indicate two different processes coinciding in frequency: the onset of the downturn of the flat bands and the onset of interband transitions in the vicinity of the $R$ point. These processes lead to additional contributions to $\sigma_{1}(v)$. The dashed green arrows correspond to the transitions between the almost parallel bands along the $M-R$ line, leading to a maximum in $\sigma_{1}(v)$. Transitions with approximately, but not exactly, the same energy (e.g., along the $\Gamma-R$ line) are responsible for smearing out this maximum and are indicated with the same arrow. The dashed blue lines are a guide to the eye. The solid purple line is an extrapolation of the effective-Hamiltonian computations from Ref. [19] to higher frequencies.

within the uncertainty, set by the experimental spectra, does not compromise it [21].

At the lowest frequencies (below approximately $2500 \mathrm{~cm}^{-1}$ or $0.3 \mathrm{eV}$ ), the interband conductivity is entirely due to transitions in the vicinity of the $\Gamma$ point. No other interband optical transitions are possible (either the direct gap between the bands is too large, or the transitions are Pauli blocked). The bands near the $\Gamma$ point are all roughly linear (two of them are basically flat), thus a linear-in-frequency interband conductivity is expected [19]. Indeed, as already noticed, $\sigma_{1}^{\text {inter }}(v)$ is proportional to frequency in this range (marked with the orange arrows). At somewhat higher $v$ ( $\left.3000-4000 \mathrm{~cm}^{-1}, 0.4-0.5 \mathrm{eV}\right)$, the flat bands start to disperse downward, thus the linearity of $\sigma_{1}^{\text {inter }}(v)$ is not expected anymore. However, the interband contributions in the vicinity of the $R$ points become allowed at roughly the same energy [cf. the two grey arrows in panel (a)]. These transitions provide a dominating contribution to conductivity, and the linear-in-frequency increase of $\sigma_{1}^{\text {inter }}(v)$ is restored with a larger slope [the grey arrow in panel (b)]. At $v \geqslant 6000 \mathrm{~cm}^{-1}(0.8 \mathrm{eV})$, the optical conductivity flattens out, forming a broad flat maximum. We attribute it to the transitions between the almost parallel bands along the $M-R$ line, shown as the dotted green arrows. The maximum is not sharp because there are many other transitions with comparable frequencies; see, e.g., the dotted green arrow between the $\Gamma$ and $R$ points. After the relatively flat region, $\sigma_{1}^{\text {inter }}(v)$ continues to rise [see Fig. 1(b)], as more and more bands get involved in optical transitions.

In Fig. 4(b), we also compare our results with the effectiveHamiltonian calculations [19] for the contributions near the $\Gamma$ point. An extrapolation of these calculations, originally performed for $v<320 \mathrm{~cm}^{-1}(40 \mathrm{meV})$, to higher frequencies is shown as a solid purple line. The experimental $\sigma_{1}^{\text {inter }}(v)$ is generally steeper than the results of these calculations. A very similar behavior of the experimental conductivity versus such effective-Hamiltonian calculations has also been reported in Ref. [11]. Perhaps, at the lowest frequencies the match between the experiment and the model is better, but our signal-to-noise ratio is not sufficient for final conclusions (one should also remember that the $\sigma_{1}^{\text {inter }}(v)$ spectrum is obtained utilizing a Drude-terms subtracting procedure). In any case, the mismatch can be related to deviations of the bands from linearity even at low energies $[4,5,20,27]$. This can be clarified in more advanced band-structure-based opticalconductivity calculations, which are beyond the scope of this paper [33].

Having established the connection between the features in the experimental low-energy interband conductivity and the band structure, we would like to add another note on the intraband response. The exact shape of the free-carrier contribution is obviously sample dependent, because, e.g., the impurity scattering rate differs from sample to sample. However, if the total amount of doping is low enough, the plasma frequency is fixed by the position of the Fermi level, which, in turn, can be found within the band structure calculation procedure. Such calculations [20] produced $\hbar \omega_{\mathrm{pl}}=1.344 \mathrm{eV}$, in excellent agrement with our result obtained above, $1.39 \pm$ $0.08 \mathrm{eV}$. Furthermore, the calculated and the observed spectral positions of the screened plasma frequency (the zero-crossing points of $\varepsilon_{1}$ ) also match each other very well, as can be seen in Fig. 3(b). This agreement of the plasma frequencies also indicates a good quality of our sample in terms of low defect and impurity concentration. 


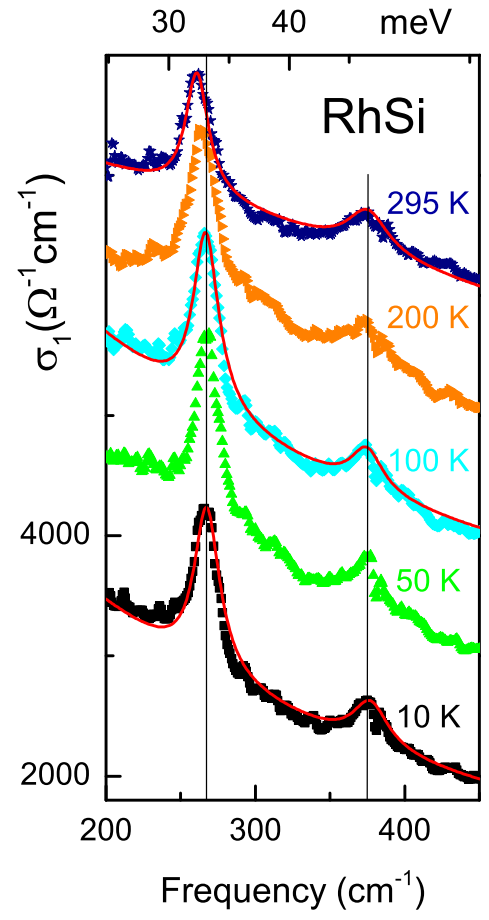

FIG. 5. Optical conductivity of $\mathrm{RhSi}$ at the frequencies near the observed phonon modes for selected temperatures. The $y$ scale corresponds to the $10-\mathrm{K}$ spectra. The spectra for other temperatures are shifted upwards for clarity. Results of Lorentzian fits of the phonon modes (on an electronic background) are shown for 10, 100, and $295 \mathrm{~K}$ as solid red lines. The black vertical lines are a guide to the eye.

\section{B. Phonons}

Based on its crystallographic symmetry, RhSi is supposed to show five infrared-active phonon modes; however, we can clearly identify only two of them in our spectra. The other modes are likely too weak to be resolved on top of the electronic background within the available experimental accuracy. The two sharp phonon modes fall in the spectral range from 200 to $400 \mathrm{~cm}^{-1}(25-50 \mathrm{meV})$; see Fig. 5(c). The positions of these modes at $T=10 \mathrm{~K}$ are marked with thin vertical lines.

Both modes can be accurately described by Lorentzians at any temperature. No asymmetric (Fano-like) models are necessary. This is in contrast to the situation in FeSian isostructural analog of $\mathrm{RhSi}$ with a presumably important role of electron correlations-where strong phonon-line asymmetry was reported in the optical spectra and related to electron-phonon coupling [34]. The absence of detectable electron-phonon coupling indicates that phonon-mediated electron-correlation effects are likely of no relevance in $\mathrm{RhSi}$.

Let us finally mention that the phonon modes observed in $\mathrm{RhSi}$ demonstrate a usual broadening as temperature rises. Additionally, the low-frequency mode shows a small softening of its central frequency with increasing $T$, which can be explained by usual thermal expansion (possible softening of another mode might be not resolved because of a larger width of this mode).

\section{CONCLUSIONS}

We have measured the broadband optical response of the multifold semimetal RhSi. Infrared-active phonons and electronic transitions are revealed in this study. The phonon modes demonstrate a trivial temperature dependence with no indications of strong electron-phonon coupling. The intraband electronic contribution (Drude) is relatively strong, with an (unscreened) plasma frequency of approximately $1.4 \mathrm{eV}$. The interband optical conductivity demonstrates a linear increase at low frequencies (below $300 \mathrm{meV}$ ). We interpret this increase as a signature of the transitions between the linear bands crossing around the $\Gamma$ point. At somewhat higher frequencies (400-600 meV), contributions from the linear bands near the $R$ point (Pauli blocked at lower frequencies) manifest themselves as an increased slope of $\sigma_{1}(\omega)$. These observations confirm the predictions for the optical-conductivity behavior in multifold semimetals.

\section{ACKNOWLEDGMENTS}

We thank Prof. Tetsuro Habe and Prof. Zhi Li for contacting us after a preprint of this paper was posted to the arXiv and for subsequent useful discussions. We are grateful to Ms. Gabriele Untereiner for valuable technical support. E.U. acknowledges support from the European Social Fund and from the Ministry of Science, Research, and the Arts of Baden-Württemberg. This work was partly supported by the Deutsche Forschungsgemeinschaft (DFG) via Grant No. DR228/51-1.
[1] J. L. Mañes, Existence of bulk chiral fermions and crystal symmetry, Phys. Rev. B 85, 155118 (2012).

[2] B. Bradlyn, J. Cano, Z. Wang, M. G. Vergniory, C. Felser, R. J. Cava, and B. A. Bernevig, Beyond Dirac and Weyl fermions: Unconventional quasiparticles in conventional crystals, Science 353, aaf5037 (2016).

[3] G. Chang, B. J. Wieder, F. Schindler, D. S. Sanchez, I. Belopolski, S.-M. Huang, B. Singh, D. Wu, T.-R. Chang, T. Neupert, S.-Y. Xu, H. Lin, and M. Z. Hasan, Topological quantum properties of chiral crystals, Nat. Mater. 17, 978 (2018).

[4] G. Chang, S.-Y. Xu, B. J. Wieder, D. S. Sanchez, S.-M. Huang, I. Belopolski, T.-R. Chang, S. Zhang, A. Bansil, H. Lin, and M. Z. Hasan, Unconventional Chiral Fermions and Large Topological Fermi Arcs in RhSi, Phys. Rev. Lett. 119, 206401 (2017).

[5] P. Tang, Q. Zhou, and S.-C. Zhang, Multiple Types of Topological Fermions in Transition Metal Silicides, Phys. Rev. Lett. 119, 206402 (2017).

[6] D. S. Sanchez, I. Belopolski, T. A. Cochran, X. Xu, J.-X. Yin, G. Chang, W. Xie, K. Manna, V. Süß, C.-Y. Huang, N. Alidoust, D. Multer, S. S. Zhang, N. Shumiya, X. Wang, G.-Q. Wang, T.-R. Chang, C. Felser, S.-Y. Xu, S. Jia, H. Lin, and M. Z. Hasan, Topological chiral crystals with helicoid-arc quantum states, Nature (London) 567, 500 (2019). 
[7] Z. Rao, H. Li, T. Zhang, S. Tian, C. Li, B. Fu, C. Tang, L. Wang, Z. Li, W. Fan, J. Li, Y. Huang, Z. Liu, Y. Long, C. Fang, H. Weng, Y. Shi, H. Lei, Y. Sun, T. Qian, and H. Ding, Observation of unconventional chiral fermions with long Fermi arcs in CoSi, Nature (London) 567, 496 (2019).

[8] N. B. M. Schröter, D. Pei, M. G. Vergniory, Y. Sun, K. Manna, F. de Juan, J. A. Krieger, V. Süß, M. Schmidt, P. Dudin, B. Bradlyn, T. K. Kim, T. Schmitt, C. Cacho, C. Felser, V. N. Strocov, and Y. Chen, Chiral topological semimetal with multifold band crossings and long Fermi arcs, Nat. Phys. 15, 759 (2019).

[9] D. Takane, Z. Wang, S. Souma, K. Nakayama, T. Nakamura, H. Oinuma, Y. Nakata, H. Iwasawa, C. Cacho, T. Kim, K. Horiba, H. Kumigashira, T. Takahashi, Y. Ando, and T. Sato, Observation of Chiral Fermions with a Large Topological Charge and Associated Fermi-arc Surface States in CoSi, Phys. Rev. Lett. 122, 076402 (2019).

[10] F. de Juan, A. G. Grushin, T. Morimoto, and J. E. Moore, Quantized circular photogalvanic effect in Weyl semimetals, Nat. Commun. 8, 15995 (2017).

[11] D. Rees, K. Manna, B. Lu, T. Morimoto, H. Borrmann, C. Felser, J. E. Moore, D. H. Torchinsky, and J. Orenstein, Observation of topological photocurrents in the chiral Weyl semimetal RhSi, arXiv:1902.03230.

[12] P. Hosur, S. A. Parameswaran, and A. Vishwanath, Charge Transport in Weyl Semimetals, Phys. Rev. Lett. 108, 046602 (2012).

[13] Á. Bácsi and A. Virosztek, Low-frequency optical conductivity in graphene and in other scale-invariant two-band systems, Phys. Rev. B 87, 125425 (2013).

[14] P. E. C. Ashby and J. P. Carbotte, Chiral anomaly and optical absorption in Weyl semimetals, Phys. Rev. B 89, 245121 (2014).

[15] R. Y. Chen, S. J. Zhang, J. A. Schneeloch, C. Zhang, Q. Li, G. D. Gu, and N. L. Wang, Optical spectroscopy study of the three-dimensional Dirac semimetal $\mathrm{ZrTe}_{5}$, Phys. Rev. B 92, 075107 (2015).

[16] B. Xu, Y. M. Dai, L. X. Zhao, K. Wang, R. Yang, W. Zhang, J. Y. Liu, H. Xiao, G. F. Chen, A. J. Taylor, D. A. Yarotski, R. P. Prasankumar, and X. G. Qiu, Optical spectroscopy of the Weyl semimetal TaAs, Phys. Rev. B 93, 121110 (2016).

[17] D. Neubauer, J. P. Carbotte, A. A. Nateprov, A. Löhle, M. Dressel, and A. V. Pronin, Interband optical conductivity of the [001]-oriented Dirac semimetal $\mathrm{Cd}_{3} \mathrm{As}_{2}$, Phys. Rev. B 93, 121202 (2016).

[18] S. Kimura, H. Yokoyama, H. Watanabe, J. Sichelschmidt, V. Süß, M. Schmidt, and C. Felser, Optical signature of Weyl electronic structures in tantalum pnictides $\mathrm{TaPn}(P n=\mathrm{P}, \mathrm{As})$, Phys. Rev. B 96, 075119 (2017).

[19] M.-Á. Sánchez-Martínez, F. de Juan, and A. G. Grushin, Linear optical conductivity of chiral multifold fermions, Phys. Rev. B 99, 155145 (2019).

[20] Z. Li, T. Iitaka, H. Zeng, and H. Su, Optical response of the chiral topological semimetal RhSi, Phys. Rev. B 100, 155201 (2019).
[21] See Supplemental Material at http://link.aps.org/supplemental/ 10.1103/PhysRevResearch.2.023018 for justification of using polished sample surfaces and for details of the Drude-term subtraction procedure.

[22] D. B. Tanner, Use of x-ray scattering functions in KramersKronig analysis of reflectance, Phys. Rev. B 91, 035123 (2015).

[23] M. Dressel and G. Grüner, Electrodynamics of Solids (Cambridge University Press, Cambridge, 2002).

[24] M. B. Schilling, A. Löhle, D. Neubauer, C. Shekhar, C. Felser, M. Dressel, and A. V. Pronin, Two-channel conduction in YbPtBi, Phys. Rev. B 95, 155201 (2017).

[25] D. Neubauer, A. Yaresko, W. Li, A. Löhle, R. Hübner, M. B. Schilling, C. Shekhar, C. Felser, M. Dressel, and A. V. Pronin, Optical conductivity of the Weyl semimetal NbP, Phys. Rev. B 98, 195203 (2018).

[26] Z. Qiu, C. Le, Z. Liao, B. Xu, R. Yang, J. Hu, Y. Dai, and X. Qiu, Observation of a topological nodal-line semimetal in $\mathrm{YbMnSb}_{2}$ through optical spectroscopy, Phys. Rev. B 100, 125136 (2019).

[27] https://www.topologicalquantumchemistry.com; http: //www.cryst.ehu.es; B. Bradlyn, L. Elcoro, J. Cano, M. G. Vergniory, Z. Wang, C. Felser, M. I. Aroyo, and B. A. Bernevig, Topological quantum chemistry, Nature (London) 547, 298 (2017); M. G. Vergniory, L. Elcoro, C. Felser, N. Regnault, B. A. Bernevig, and Z. Wang, A complete catalogue of high-quality topological materials, ibid. 566, 480 (2019).

[28] F. Hütt, A. Yaresko, M. B. Schilling, C. Shekhar, C. Felser, M. Dressel, and A. V. Pronin, Linear-in-Frequency Optical Conductivity in GdPtBi due to Transitions near the Triple Points, Phys. Rev. Lett. 121, 176601 (2018).

[29] R. Kemmler, R. Hübner, A. Löhle, D. Neubauer, I. Voloshenko, L. M. Schoop, M. Dressel and A. V. Pronin, Free-carrier dynamics in $\mathrm{Au}_{2} \mathrm{~Pb}$ probed by optical conductivity measurements, J. Phys.: Condens. Matter 30, 485403 (2018).

[30] A. J. Frenzel, C. C. Homes, Q. D. Gibson, Y. M. Shao, K. W. Post, A. Charnukha, R. J. Cava, and D. N. Basov, Anisotropic electrodynamics of type-II Weyl semimetal candidate $\mathrm{WTe}_{2}$, Phys. Rev. B 95, 245140 (2017).

[31] D. Chaudhuri, B. Cheng, A. Yaresko, Q. D. Gibson, R. J. Cava, and N. P. Armitage, Optical investigation of the strong spinorbit-coupled magnetic semimetal $\mathrm{YbMnBi}_{2}$, Phys. Rev. B 96, 075151 (2017).

[32] D. Grassano, O. Pulci, A. M. Conte, F. Bechstedt, Validity of Weyl fermion picture for transition metals monopnictides TaAs, TaP, NbAs, and NbP from ab initio studies, Sci. Rep. 8, 3534 (2018).

[33] An elaborative theoretical study of the optical conductivity of $\mathrm{CoSi}$-another compound from the same multifold familywas recently published: T. Habe, Dynamical conductivity in the multiply degenerate point-nodal semimetal CoSi, Phys. Rev. B 100, 245131 (2019).

[34] A. Damascelli, K. Schulte, D. van der Marel, and A. A. Menovsky, Infrared spectroscopic study of phonons coupled to charge excitations in FeSi, Phys. Rev. B 55, R4863(R) (1997). 